\title{
Efficacy and Safety of Continuous Hemodiafiltration for Acute Decompensated Heart Failure
}

\author{
Hiroki Shiomi, ${ }^{1}$ MD, Kinya Matsubara, ${ }^{1}$ MD, Makoto Ariyoshi, ${ }^{1}$ MD, Yumi Iwamura, ${ }^{1}$ MD, \\ Kensuke Fukui, ${ }^{1}$ MD, Tomoko SaKamoto, ${ }^{1}$ MD, Takayoshi Sawanishi, ${ }^{1}$ MD, Masami Shima, ${ }^{1}$ MD, \\ and Keizo Furukawa, ${ }^{1}$ MD
}

\begin{abstract}
SUMMARY
The mortality of heart failure patients with renal insufficiency is high, and these patients tend to develop diuretic resistance. Under these conditions, continuous hemodiafiltration (CHDF) is a possible alternative volume reduction therapy to diuretics. However, its efficacy and safety are not clear. Between April 2005 and March 2008, 248 patients with acute decompensated heart failure were admitted to the CCU of Kyoto City Hospital. Of those patients, 31 (20 volume overloaded heart failure, 11 cardiogenic shock) received CHDF therapy, and their weight loss, acute hemodynamic changes, and clinical outcome were assessed to evaluate the efficacy and safety of CHDF therapy. CHDF was performed for $6.5 \pm 6.5$ days. There was no significant change in acute hemodynamics after CHDF initiation. In the volume overloaded heart failure $(\mathrm{VH})$ group, significant weight loss was observed at 24 hours and 48 hours after CHDF initiation $(P<0.001)$. In-hospital mortality of the VH group and cardiogenic shock $(\mathrm{CS})$ group were $10.0 \%$ and $54.5 \%$, respectively. CHDF for acute decompensated heart failure (ADHF) is a safe, effective, and reliable volume reduction therapy for volume overloaded heart failure. Further investigation is required to assess the effectiveness of CHDF for cardiogenic shock. (Int Heart J 2010; 51: 247-251)
\end{abstract}

Key words: Heart failure, CHDF, Cardiogenic shock

$\mathrm{M}$ any previous studies have demonstrated that renal insufficiency is associated with poor prognosis in patients with heart failure (HF), ${ }^{1-4)}$ and that $25-30 \%$ of patients with HF develop diuretic resistance. ${ }^{5)}$ Under these conditions, ultrafiltration (UF) has been shown to be an alternative volume reduction therapy for diuretics, and a continuous ultrafiltration method has been recommended because of its safety for hemodynamics. ${ }^{6,7)}$ It has been reported that continuous hemodiafiltration (CHDF) is also a possible alternative volume reduction therapy for diuretics and is being anticipated as a method for cytokine excision. ${ }^{8)}$ However, there is not enough data available on its efficacy and safety. We therefore conducted this study to evaluate the efficacy and safety of CHDF therapy for acute decompensated heart failure (ADHF).

\section{Methods}

Patient population: Between April 2005 and March 2008, 248 patients with acute decompensated heart failure were admitted to the CCU of Kyoto City Hospital. Of these patients, 39 received CHDF therapy. They received continuous blood purification due to their hemodynamic instability and the possibility of unknown factors threatening their hemodynamics in a situation where their condition would acutely worsen. To clarify the efficacy of CHDF therapy, we adopted the following inclusion criteria: 1) CHDF initiation within 24 hours after admission or the time at which congestive heart failure (CHF) acutely worsened in patients already admitted, and 2) To continue CHDF therapy over 6 hours. Exclusion criteria were 1) the use of CHDF for electrolyte disorders, 2) the use of CHDF in order to remove contrast medium after percutaneous coronary intervention (PCI), or 3) the use of CHDF for acute renal failure (ARF) induced by severe sepsis or any other conditions which were not CHF. Thirty-one patients were enrolled and divided into 2 groups: a volume overloaded heart failure (VH) group and a cardiogenic shock (CS) group. The CS group was defined as CHF patients with systolic blood pressure $\leqq 90 \mathrm{mmHg}$ or reduced by $20 \%$ compared to usual. The VH group was defined as CHF patients without cardiogenic shock. Eight patients (1 hyperkalemia, 3 ARF induced by severe sepsis, 4 use of removal contrast medium after PCI) were excluded. CHF was diagnosed by 2 or more cardiologists using the Framingham criteria. Information regarding demographic and clinical characteristics, medical history, management strategies, therapeutic effects, and clinical outcomes was collected from medical records.

CHDF procedure: CHDF therapy was performed using a JUN- $600^{\circledR}$ or JUN-55X ${ }^{\circledR}$ bedside console. Veno-venous double lumen catheters were placed in the internal jugular or femoral veins for vascular access, and polysulphone filters (HEMOFEEL $^{\circledR}$ ) or polymethylmethacrylate filters (HEMOFEEL ${ }^{\circledR}$ )

From the ${ }^{1}$ Department of Cardiology, Kyoto City Hospital, Kyoto, Japan.

Address for correspondence: Hiroki Shiomi, MD, Department of Cardiology, Kyoto City Hospital, 1-2 Mibu Higashi Tagada-cho, Nakagyo-ku, Kyoto 604-8845, Japan.

Received for publication December 16, 2009

Revised and accepted April 12, 2010. 
were used as hemofilters. Blood flow was set at $50-120 \mathrm{~mL} /$ hour and the fluid removal rate was no more than $500 \mathrm{~mL} /$ hour. Patients who received CHDF therapy were treated with nafamostat mesilate to maintain their activated clotting time between 150 and 200 seconds (in the postfilter line) during CHDF therapy.

Assessments and endpoints: Changes in heart rate (HR) and blood pressure (BP) were assessed in both the VH group and $\mathrm{CS}$ group before CHDF initiation, and at 6 and 24 hours after $\mathrm{CHDF}$ initiation to evaluate the safety of CHDF therapy for acute decompensated heart failure (ADHF). Urination, water removal to CHDF, and net fluid loss 6 hours, 24 hours, and 48 hours after CHDF initiation were assessed as was weight change before and 24 and 48 hours after CHDF initiation for evaluation of the efficacy of CHDF therapy for ADHF. We followed-up the duration of CCU stay, hospitalization, 28-day mortality, in-hospital mortality, and 12 month mortality as a clinical outcome.

Statistical analysis: Continuous variables are expressed as the mean \pm SD or median. Dichotomous variables are expressed as percentages. Statistical comparisons of continuous variables were performed using Student's paired $t$-test or the Wilcoxon rank-sum test as appropriate, and $P$ values less than 0.05 were considered statistically significant. All statistical analyses were performed with Stat View software version 5.0 (SAS Institute Inc, Cary, NC, USA).

\section{RESULTS}

Patient characteristics: The patient characteristics are outlined in Table I. The study group comprised 31 patients, and 20 were allocated to the VH group and 11 to the CS group. New York Heart Association (NYHA) functional class IV was present in 95.0\% (19/20) of patients even in the VH group. In the CS group, return of spontaneous circulation (ROSC) after cardiopulmonary arrest was present in $54.5 \%$ of patients $(6 / 11)$, and percutaneous cardiopulmonary support (PCPS) was used in $54.5 \%(6 / 11)$

CHDF therapy: CHDF was initiated within $4.1 \pm 4.4$ hours after hospitalization or the time at which CHF acutely worsened. Average duration of CHDF therapy was $6.5 \pm 6.5$ days (Table IV). The mean effluent flow rate was $1852 \pm 711 \mathrm{~mL} /$ hour (corresponding to $34.5 \pm 15.5 \mathrm{~mL} / \mathrm{kg} /$ hour).

Acute hemodynamic changes after CHDF initiation are shown in Table II. The changes in HR and BP 6 hours and 24 hours after CHDF initiation were not significant in the total patient population (Figure 1). In the CS group, diastolic pressure (DSP) was increased $(P: 0.03)$ and HR was decreased $(P$ : 0.01) 24 hours after CHDF initiation, but neither had a negative influence on hemodynamics.

Fluid removal and weight loss during CHDF therapy are shown in Table III. In the VH group, net fluid loss was significant at 6 hours $(-915.6 \pm 694.0 \mathrm{~mL} ; P<0.001), 24$ hours $(-1883.5 \pm 1362.6 \mathrm{~mL} ; P<0.001)$, and 48 hours $(-2040 \pm$

Table I. Clinical Characteristics of Study Patients

\begin{tabular}{|c|c|c|c|}
\hline & VH group $(n=20)$ & CS group $(n=11)$ & Total $(n=31)$ \\
\hline Age (years) & $70.3 \pm 14.3$ & $65.3 \pm 9.3$ & $68.5 \pm 13.0$ \\
\hline Gender (male/female) & $12 / 8$ & $7 / 4$ & $12 / 19$ \\
\hline NYHA class (III/IV) & $1 / 19$ & $0 / 11$ & $1 / 30$ \\
\hline Killip class (III/IV) & $20 / 0$ & $0 / 11$ & $20 / 11$ \\
\hline Cardio-pulmonary arrest & 0.0 & 54.5 & 19.4 \\
\hline IABP & 0.0 & 54.5 & 19.4 \\
\hline $\operatorname{LVEF}(\%)$ & $50.7 \pm 13.8$ & $46.7 \pm 13.4$ & $49.3 \pm 13.8$ \\
\hline Prior heart failure $(\%)$ & 60.0 & 9.0 & 41.9 \\
\hline \multicolumn{4}{|l|}{ Etiology of CHF } \\
\hline Coronary artery disease $(\%)$ & 75.0 & 90.9 & 80.6 \\
\hline Cardiomyopathy (\%) & 15.0 & 9.1 & 12.9 \\
\hline Valvular disease $(\%)$ & 40.0 & 9.1 & 29.0 \\
\hline Hypertensive heart disease $(\%)$ & 70.0 & 27.3 & 54.8 \\
\hline \multicolumn{4}{|l|}{ Comorbidities } \\
\hline History of hypertension (\%) & 90.0 & 81.8 & 87.1 \\
\hline Dyslipidemia $(\%)$ & 45.0 & 63.6 & 51.6 \\
\hline Diabetes mellitus (\%) & 40.0 & 27.3 & 48.4 \\
\hline Atrial fibrillation (\%) & 25.0 & 18.2 & 22.6 \\
\hline Endstage renal disease (\%) & 30.0 & 18.2 & 25.8 \\
\hline \multicolumn{4}{|l|}{ Medication } \\
\hline$\beta$-Blocker $(\%)$ & 50.0 & 0.0 & 32.3 \\
\hline ACE-I/ARB (\%) & 75.0 & 18.2 & 51.6 \\
\hline Diuretics $(\%)$ & 50.0 & 18.2 & 38.7 \\
\hline Catecholamines use $>5 \gamma(\%)$ & 0.0 & 90.9 & 32.3 \\
\hline Serum hemoglobin $(\mathrm{g} / \mathrm{dL})$ & $9.8 \pm 2.5$ & $10.4 \pm 2.6$ & $10.0 \pm 2.6$ \\
\hline Serum creatinine $(\mathrm{mg} / \mathrm{dL})$ & $4.66 \pm 3.01$ & $3.00 \pm 2.48$ & $4.07 \pm 2.94$ \\
\hline Estimated GFR $\left(\mathrm{mL} \cdot \mathrm{min}^{-1} \cdot 1.73 \mathrm{~m}^{-2}\right)$ & $18.7 \pm 19.5$ & $33.8 \pm 27.2$ & $24.1 \pm 23.3$ \\
\hline
\end{tabular}

Values are mean \pm SD. HV group indicates high volume heart failure group; CS group, cardiogenic shock group; IABP, intra aortic balloon pumping; NYHA, New York Heart Association; LVEF, LV ejection fraction; ACE-I, angiotensin converting enzyme-inhibitor; ARB, angiotensin II receptor blocker; and GFR, glomerular filtration rate calculated by Cockcroft-Gault equation. 
Table II. Changes in Hemodynamics After CHDF Initiation

\begin{tabular}{|c|c|c|c|c|}
\hline & \multicolumn{4}{|c|}{ HV group $(n=20)$} \\
\hline & Before & 6 hours & 24 hours & $P$ \\
\hline Heart rate (beats/minute) & $83.0 \pm 11.6$ & $87.0 \pm 14.1$ & $84.0 \pm 12.7$ & NS/NS \\
\hline \multicolumn{5}{|l|}{ Blood pressure (mmHg) } \\
\hline Sytolic pressure & $128.8 \pm 23.7$ & $129.2 \pm 23.1$ & $116.4 \pm 18.7$ & NS/NS \\
\hline \multirow[t]{3}{*}{ Diastolic pressure } & $55.4 \pm 13.7$ & $58.2 \pm 19.0$ & $51.8 \pm 13.0$ & NS/NS \\
\hline & \multicolumn{4}{|c|}{ CS group $(n=11)$} \\
\hline & Before & 6 hours & 24 hours & $P$ \\
\hline Heart rate (beats/minute) & $106.5 \pm 23.6$ & $97.8 \pm 12.8$ & $86.3 \pm 10.2$ & $\mathrm{NS} / 0.01$ \\
\hline \multicolumn{5}{|l|}{ Blood pressure (mmHg) } \\
\hline Sytolic pressure & $105.0 \pm 23.8$ & $112.2 \pm 24.8$ & $109.2 \pm 15.3$ & NS/NS \\
\hline \multirow[t]{3}{*}{ Diastolic pressure } & $53.9 \pm 8.9$ & $55.5 \pm 11.1$ & $61.1 \pm 13.2$ & $\mathrm{NS} / 0.03$ \\
\hline & \multicolumn{4}{|c|}{ Total $(n=31)$} \\
\hline & Before & 6 hours & 24 hours & $P$ \\
\hline Heart rate (beats/minute) & $91.3 \pm 20.3$ & $90.8 \pm 14.6$ & $84.8 \pm 11.9$ & NS/NS \\
\hline \multicolumn{5}{|l|}{ Blood pressure (mmHg) } \\
\hline Sytolic pressure & $120.3 \pm 26.3$ & $123.1 \pm 25.1$ & $113.8 \pm 17.9$ & NS/NS \\
\hline Diastolic pressure & $54.9 \pm 12.2$ & $57.2 \pm 16.7$ & $55.1 \pm 13.8$ & NS/NS \\
\hline
\end{tabular}

Values are mean $\pm \mathrm{SD}$

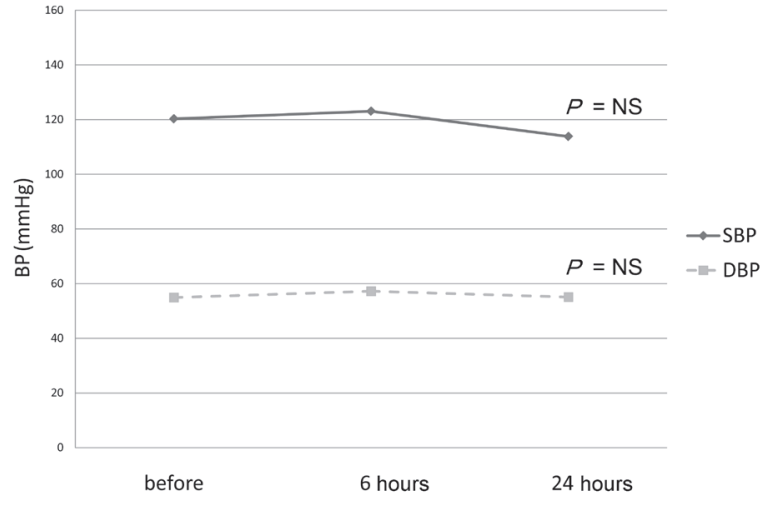

Figure 1. Hemodynamic change after CHDF initiation. Blood pressure (BP) 6 hours and 24 hours after CHDF initiation did not change significantly compared with that of CHDF initiation in the total combined patient population.

$1604.4 \mathrm{~mL} ; P<0.001)$ after CHDF initiation. Weight loss was also significant at 24 hours $(-2.1 \pm 1.3 \mathrm{~kg} ; P<0.001)$ and 48 hours $(-2.8 \pm 1.7 \mathrm{~kg} ; P<0.001$, Figure 2$)$. Net fluid loss and weight change in the CS group were not analyzed because treatment for those who are in Forrester IV is not aimed at reducing volume, so it is inappropriate to evaluate the efficacy of $\mathrm{CHDF}$ as a volume reduction therapy under these conditions.

Clinical outcome and adverse events: Clinical outcome and adverse events in CHDF therapy are shown in Table IV. Hospitalization of the $\mathrm{VH}$ group, $\mathrm{CS}$ group and total combined population were $46.5 \pm 24.1,54.7 \pm 55.2$, and $46.5 \pm 24.1$ days, respectively. The duration of CCU stay of the VH group, CS group, and total combined population was $9.7 \pm 18.1,16.8 \pm$ 8.3 , and $12.2 \pm 15.7$ days, while in-hospital mortality was

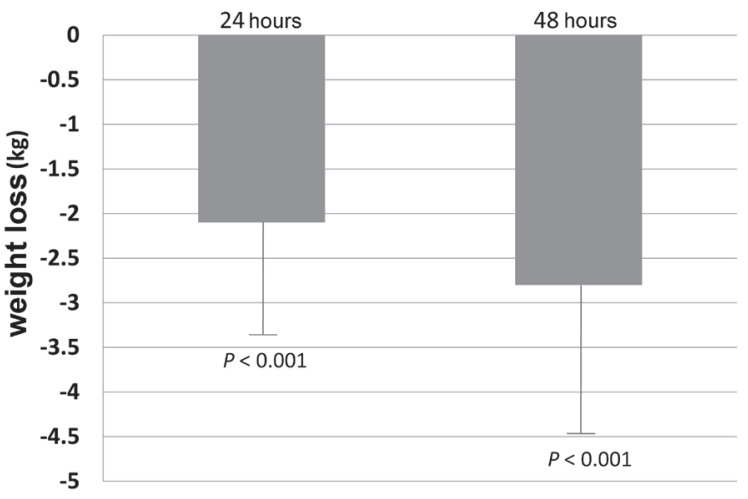

Figure 2. Weight change after CHDF initiation. Weight loss after CHDF initiation was significant at 24 hours $(-2.1 \pm 1.3 \mathrm{~kg} ; P<0.001)$ and 48 hours $(-2.8 \pm 1.7 \mathrm{~kg} ; P<0.001)$.

$10.0 \%, 54.5 \%$ and $25.8 \%$, respectively. Twelve-month mortality was $10.0 \%, 54.5 \%$, and $25.8 \%$, respectively. CHF re-admission and 28-day mortality are also shown in Table IV.

Possible complications of CHDF therapy are catheter-related infection, bleeding, and hypotension. In the present study, two bleeding patients were observed in the CS group, one due to colon cancer and another due to a duodenum ulcer. One patient with hypotension in the VH group was administered catecholamine to stabilize blood pressure during CHDF. No electrolyte disorders such as hypokalemia were observed because electrolytes were monitored and corrected every 4 to 6 hours during CHDF. 
Table III. Results of CHDF Therapy

\begin{tabular}{lccccc}
\hline & & \multicolumn{3}{c}{ HV group $(n=20)$} \\
& Before & 6 hours & 24 hours & 48 hours \\
\hline Urine output $(\mathrm{mL})$ & - & $-321.3 \pm 373.7$ & $-919.7 \pm 1078.8$ & $-1661.8 \pm 1995.4$ \\
Fluid removal by CHDF $(\mathrm{mL})$ & - & $-917.2 \pm 573.1$ & $-2090.6 \pm 1114.3$ & $-2514.6 \pm 1524.1$ & - \\
Net fluid loss $(\mathrm{mL})$ & - & $-915.6 \pm 694.0$ & $-1883.5 \pm 1362.6$ & $-2040.2 \pm 1604.4$ & $<0.001 /<0.001<0.001$ \\
Weight $(\mathrm{kg})$ & $56.7 \pm 13.6$ & - & $54.6 \pm 13.6$ & $53.9 \pm 13.3$ & $<0.001 /<0.001$ \\
Weight change $(\mathrm{kg})$ & - & - & $-2.1 \pm 1.3$ & $-2.8 \pm 1.7$ \\
\hline
\end{tabular}

Table IV. Clinical Results and Outcome

\begin{tabular}{lccc}
\hline & HV group $(n=20)$ & CS group $(n=11)$ & Total $(n=31)$ \\
\hline Hospitalization (days) & $46.5 \pm 24.1$ & $54.7 \pm 55.2$ & $46.5 \pm 24.1$ \\
CCU stay (days) & $9.7 \pm 18.1$ & $16.8 \pm 8.3$ & $12.2 \pm 15.7$ \\
Duration of CHDF (days) & $3.9 \pm 5.8$ & $11.2 \pm 4.9$ & $6.5 \pm 6.5$ \\
Adverse events associated with CHDF & & & \\
$\quad$ Catheter-related infection & $0 \%(0 / 20)$ & $0 \%(0 / 11)$ & $0 \%(0 / 31)$ \\
$\quad$ Bleeding & $0 \%(0 / 20)$ & $18 \%(2 / 11)$ & $6.5 \%(2 / 31)$ \\
$\quad$ Hypotension & $5.0 \%(1 / 20)$ & $0 \%(0 / 11)$ & $3.2 \%(1 / 31)$ \\
In-hospital mortality & $10.0 \%(2 / 20)$ & $54.5 \%(6 / 11)$ & $25.8 \%(8 / 31)$ \\
28-day mortality & $5.0 \%(1 / 20)$ & $27.3 \%(3 / 11)$ & $13.0 \%(4 / 31)$ \\
CHF readmission within 6 months & $25.0 \%(5 / 20)$ & $0 \%(0 / 11)$ & $16.1 \%(5 / 31)$ \\
12-month mortality & $10.0 \%(2 / 20)$ & $54.5 \%(6 / 11)$ & $25.8 \%(8 / 31)$ \\
\hline
\end{tabular}

Values are mean $\pm \mathrm{SD}$.

\section{Discussion}

Many previous studies have demonstrated that renal insufficiency is associated with poor prognosis in patients with $\mathrm{HF}$, and that renal insufficiency and diuretic resistance have emerged as problems in CHF treatment. ${ }^{1-4)}$ On the other hand, therapy for patients with ADHF should aim to achieve euvolemia, because most patients with ADHF have symptoms of volume overload. ${ }^{9)}$ Based on this background, alternative volume reduction therapy for diuretics is needed, and previous studies have shown that ultrafiltration therapy is a safe and efficacious alternative volume reduction therapy for $\mathrm{CHF}^{6,10-12)}$ CHDF therapy has also been reported to be effective in the treatment of $\mathrm{CHF}^{8)}$ Furthermore, CHDF is being anticipated as a superior method for cytokine excision ${ }^{8)}$ compared with ultrafiltration. Cytokine excision may be effective in heart failure treatment. However, there is not enough data available on its safety and efficacy. We therefore conducted the present study to evaluate the safety and efficacy of CHDF therapy for acute decompensated heart failure (ADHF).

In the present study, the changes in $\mathrm{HR}$ and $\mathrm{BP}$ before and after CHDF initiation were assessed in order to evaluate the safety of CHDF therapy for ADHF. Neither HR nor BP changed significantly, and no obvious negative influence of $\mathrm{CHDF}$ on hemodynamics was observed. This result is in agreement with previous studies that reported the safety of acute blood purification for ADHF. Although CHDF might have been considered as a contraindication for patients with cardiogenic shock, our results suggest that CHDF therapy was safe for the CS group. This indicates CHDF therapy is a possible treatment for critically ill patients with cardiogenic shock for which blood purification could be considered a contraindica- tion.

We assessed net fluid loss and weight change before and after CHDF in the VH group to evaluate the efficacy of CHDF as an alternative volume reduction therapy for diuretics. The efficacy of CHDF therapy for ADHF was confirmed in the VH group, as significant net fluid loss and weight loss were found after CHDF initiation. Net fluid loss and weight change in the CS group were not analyzed because treatment for those who were in Forrester IV is not aimed at reducing volume, so it is inappropriate to evaluate the efficacy of CHDF as a volume reduction therapy under these conditions. The purpose of CHDF for use in patients with cardiogenic shock is not only volume reduction but also renal support for complicated renal failure and cytokine excision. This is because it has been reported that the cytokines IL- 6 and TNF- $\alpha$ are increased in acute heart failure, and these cytokines result in the poor prognosis of CHF. ${ }^{13)}$ This is the reason why we selected CHDF, not ultrafiltration, for $\mathrm{ADHF}$ in both the $\mathrm{VH}$ and $\mathrm{CS}$ groups.

In the present study, in-hospital mortality of the $\mathrm{VH}$ and CS group was $10.0 \%$ and $54.5 \%$, respectively. These results are equivalent to the prognosis of $\mathrm{CHF}$ patients $(11.3-27 \%)$ and cardiogenic shock patients (50-80\%), according to previous reports. ${ }^{1,14-18)}$ But these results could not be easily compared to this data because of the limited number in the present study and the differences in patient characteristics. To evaluate the efficacy other than the volume reduction associated with CHDF therapy, it is necessary to compare the effects of cardiac function and mortality of CHDF therapy with ultrafiltration. Therefore, discussion of this point is not appropriate in the present study.

The safety and efficacy of acute blood purification for $\mathrm{ADHF}$ as an alternative volume reduction therapy for diuretics 
in conditions of renal insufficiency and diuretic resistance have been determined. However, further investigation is required to evaluate which should be selected as an acute blood purification method: ultrafiltration or CHDF, as well as the effects on cardiac remodeling, or the timing of blood purification initiation.

Study limitations: The present study has the inherent limitations of a retrospective design and a relatively small number of patients in a single center. This study did not have a comparative group of patients who were not undergoing CHDF therapy. Thus, it is not appropriate to discuss major clinical outcomes such as prognosis or CHF rehospitalization because it would not exclude unexpected confounding factors.

However, it is important for the evaluation of CHDF therapy to accumulate pilot studies like the present study, because the study population comprises critically ill patients requiring intensive care in a CCU or ICU, and it is difficult to conduct randomized controlled studies with these patients.

It is hoped that the accumulation of pilot studies like the present study will develop into a large prospective study of CHDF therapy for ADHF.

Conclusion: Continuous hemodiafiltration (CHDF) in patients with acute decompensated heart failure (ADHF) is safe, and is an effective and reliable volume reduction therapy in volume overloaded heart failure. Further investigation is required to assess the effectiveness of CHDF for cardiogenic shock.

\section{REFERENCES}

1. Kawashiro N, Kasanuki H, Ogawa H, et al. Clinical characteristics and outcome of hospitalized patients with congestive heart failure results of the HIJC-HF registry. Circ J 2008; 72: 2015-20.

2. McClellan WM, Flanders WD, Langston RD, Jurkovitz C, Presley R. Anemia and renal insufficiency are independent risk factors for death among patients with congestive heart failure admitted to community hospitals: a population-based study. J Am Soc Nephro 2002; 13:1928-36.

3. Go AS, Chertow GM, Fan D, McCulloch CE, Hsu CY. Chronic kidney disease and the risks of death, cardiovascular events, and hospitalization. N Engl J Med 2004; 351:1296-305.

4. Hillege HL, Nitsch D, Pfeffer MA, et al. Renal function as a pre- dictor of outcome in a broad spectrum of patients with heart failure. Circulation 2006; 113: 671-8.

5. Ellison DH. Diuretic therapy and resistance in congestive heart failure. Cardiology 2001; 96: 132-43.

6. Costanzo MR. Ultrafiltration in the management of heart failure. Curr Opin Crit Care 2008; 14: 524-30. (Review)

7. Canaud B, Cristol JP, Klouche K, et al. Slow continuous ultrafiltration: a means of unmasking myocardial functional reserve in end stage cardiac disease. Contrib Nephrol 1991; 93: 79-85.

8. Nakanishi K, Hirasawa H, Sugai T, et al. Efficacy of continuous hemodiafiltration for patients with congestive heart failure. Blood Purif 2002; 20: 342-8.

9. Adams KF Jr, Fonarow GC, Emerman CL, et al. Characteristics and outcomes of patients hospitalized for heart failure in the United States: rationale, design, and preliminary observations from the first 100,000 cases in the Acute Decompensated Heart Failure National Registry (ADHERE). Am Heart J 2005; 149: 209-16.

10. Costanzo MR, Guglin ME, Saltzberg MT, et al. Ultrafiltration versus intravenous diuretics for patients hospitalized for acute decompensated heart failure. J Am Coll Cardiol 2007; 49: 675-83.

11. Costanzo MR, Saltzberg M, O’Sullivan J, Sobotka P. Early ultrafiltration in patients with acute decompensated heart failure and diuretic resistance. J Am Coll Cardiol 2005; 46: 2047-51.

12. Rogers HL, Marshall J, Bock J, et al. A randomized, controlled trial of the renal effects of ultrafiltration as compared to furosemide in patients with acute decompensated heart failure. J Cardiac Fail 2008; 14: 1-5.

13. Deswal A, Petersen NJ, Feldman AM, Young JB, White BG, Mann DL. Cytokines and cytokine receptors in advanced heart failure: an analysis of the cytokine database from the Vesnarinone trial (VEST). Circulation 2001; 103: 2055-9.

14. Ruiz-Bailén M, Rucabado-Aguilar L, Expósito-Ruiz M, et al. Cardiogenic shock in acute coronary syndrome. Med Sci Monit 2009; 15: RA57-66. (Review)

15. Hollenberg SM, Kavinsky CJ, Parrillo JE. Cardiogenic shock. Ann Intern Med 1999; 131: 47-59. (Review)

16. Sanborn TA, Feldman T. Management strategies for cardiogenic shock. Curr Opin Cardiol 2004; 19: 608-12. (Review)

17. Zannad F, Adamopoulos C, Mebazaa A, Gheorghiade M. The challenge of acute decompensated heart failure. Heart Fail Rev 2006; 11: 135-9. (Review)

18. Zannad F, Mebazaa A, Juillière Y, et al. Clinical profile, contemporary management and one-year mortality in patients with severe acute heart failure syndromes: The EFICA study. Eur J Heart Fail 2006; 8: 697-705. 